\title{
Les arts de la ville dans la prospective urbaine
}

Mise en perspective et bilan des travaux d'un colloque international

\section{Pascal Sanson}

\section{(2) OpenEdition}

\section{Journals}

Édition électronique

URL : http://journals.openedition.org/communicationorganisation/400

DOI : 10.4000/communicationorganisation.400

ISSN : 1775-3546

\section{Éditeur}

Presses universitaires de Bordeaux

\section{Édition imprimée}

Date de publication : 1 décembre 2007

Pagination : 211-221

ISSN : 1168-5549

\section{Référence électronique}

Pascal Sanson, "Les arts de la ville dans la prospective urbaine ", Communication et organisation [En ligne], 32 | 2007, mis en ligne le 01 décembre 2010, consulté le 21 septembre 2020. URL : http:// journals.openedition.org/communicationorganisation/400 ; DOI : https://doi.org/10.4000/ communicationorganisation.400

Ce document a été généré automatiquement le 21 septembre 2020.

(C) Presses universitaires de Bordeaux 


\title{
Les arts de la ville dans la prospective urbaine
}

\author{
Mise en perspective et bilan des travaux d'un colloque international
}

\author{
Pascal Sanson
}

\section{NOTE DE L'ÉDITEUR}

Débat public et médiation. Organisé par le CEDPM et la MSH « Villes et territoires » dans le cadre de la Semaine de la ville 2006 de Tours

1 Au sein des contextes bâtis, de nombreux arts visuels, la sculpture notamment, sont très souvent liés ou même complètement intégrés aux compositions architecturales. De plus, de nombreux artistes vont être à la fois architecte, peintre, sculpteur et designer en exerçant, parfois simultanément, plusieurs de ces arts du dessin. Citons par exemple, pour l'Italie à partir de la Renaissance, Le Bernin, Brunelleschi, Michel-Ange ou Sansovino et, pour la France, Jean Goujon ou Pierre Puget. Plus près de notre époque, cette tradition d'un art total de l'habitat a été remise à l'honneur par le Bauhaus et les autres protagonistes du Mouvement moderne dont Le Corbusier et Robert Mallet-Stevens.

2 Un des faits majeurs de l'évolution du postmodernisme dans le champ architectural est la redécouverte du sens de l'architecture et, plus généralement, de tout aménagement spatial. Il en a résulté un rejet du fonctionnalisme réducteur et, depuis le dernier quart $\mathrm{du} \mathrm{XX}^{\mathrm{e}}$ siècle, un incontestable renouveau de l'architecture et des arts de la ville ${ }^{1}$. Ce phénomène des architectures redevenues signifiantes s'est développé à l'échelle mondiale, conjointement à la réinvention de la sculpture publique et de l'art des jardins qui contribuent à l'aménagement des villes contemporaines. Il est donc important de comprendre l'incidence de ces créations sur la signification des paysages architecturaux et urbains. C'est la raison d'être d'une série de rencontres scientifiques dont nous avons été à l'origine et qui ont été initiées par le colloque international ${ }^{2}$ organisé par le groupe Eidos et l'association InformUrba, sous l'égide de 
l'Association Internationale de Sémiotique Visuelle. Il s'est prolongé par des échanges et des séminaires qui ont conduit aux textes rassemblés dans une publication récente ${ }^{3}$. Nous reprenons les termes d'une partie de l'exposé des motifs, initiateur de ces rencontres.

Le concept de paysage urbain se fonde dans la représentation de la ville : il procède d'une redécouverte des dimensions signifiantes des espaces habités. Par conséquent, l'histoire du paysage urbain est liée à une histoire des différents types de représentation de la ville. Inscrite dans son développement spatial, la ville se donne à voir dans son architecture, dans ses monuments, dans ses aménagements paysagers, dans les représentations dont elle fait l'objet sous la forme d'œuvres d'art; en particulier, la représentation de la ville accompagne, en lui donnant sa consistance, l'invention de la perspective entre le Moyen Âge et la Renaissance; elle se donne à penser sous la forme de discours et de philosophies et sous la forme de planification; elle se donne enfin à connaître sous la forme de toutes les sciences de la ville.

Dans notre contribution (p. 293), nous pouvions déclarer que pendant des siècles le paysage urbain avec ses monuments, ses éléments d'urbanisme, ses places, ses ponts et ses grands tracés, mais aussi avec ses architectures prestigieuses ou vernaculaires a inspiré les peintres et les graveurs. Le petit pan de mur jaune de la Vue de Delft de Vermeer, si cher à Marcel Proust, est là pour nous le rappeler, avec de plus, par la même occasion, sa place dans l'imaginaire des écrivains. C'est en effet l'investissement sémantique de ces formes urbaines qui est décrit en termes poétiques par des écrivains comme Julien Gracq (1990), Walter Benjamin (1989) ou Pierre Sansot (1984). C'est bien le paysage urbain d'une forte qualité signifiante, en prise avec l'art de tous les temps mais notamment de son temps, qui crée l'identité urbaine elle-même porteuse de lien social. L'élucidation du sens de ce paysage urbain a motivé, dès 1996, l'organisation de sessions dans le cadre du VI ${ }^{e}$ Congrès de l'A.I.S. (Association Internationale de Sémiotique), intitulées: Sémiotique du paysage urbain (Guadalajara, Mexique). La problématique a été poursuivie dans le colloque de Blois et des séminaires qui ont abouti à la publication de l'ouvrage aux Editions L'Harmattan (Cf. note 3).

\section{Historique et rattachements}

\section{Historique de la réinvention de l'art public}

Dans les années 1930, il faut saluer l'action anticipatrice de Jean Zay, après son arrivée au ministère de l'Éducation nationale pour constater les premiers signes avantcoureurs d'un retour de l'art dans l'espace public. Cette action est mise en œuvre avec l'aide de Jean Cassou, de Georges Huisman et de Louis Hautecœur. Le ministre relance la commande publique et encourage une rupture avec la tradition monumentale qui a marqué tout le XIX ${ }^{e}$ siècle. La contrainte du programme trop strict est abandonnée, les commandes peuvent être ouvertes à des artistes exclus de l'académie et l'État tente, en réaffirmant soutien à la création vivante, de réconcilier le public avec l'art de son temps. La réalisation de l'architecture intérieure du paquebot Normandie, mis à l'eau en 1932, va permettre à de nombreux artistes modernes de faire de ce navire un musée flottant: Jean Chauvin participe à la décoration du paquebot en réalisant une fontaine lumineuse destinée à éclairer la piscine, René Lalique crée des panneaux de verre et les éclairages de la salle à manger du paquebot, Georges Lepape conçoit des panneaux au 
motif géométrique inspiré par une corne d'abondance. Roger-Henri Expert, architecte des superstructures du Normandie, est aussi l'auteur avec André Granet des bassins créés dans les jardins du palais de Chaillot, éléments paysagers importants du site complètement restructuré dans son architecture par les architectes Jacques Carlu, Louis Boileau et Léon Azema pour l'Exposition universelle de Paris.

En 1937, cette Exposition, fameuse pour la confrontation architecturale des deux dictatures régnant sur l'Est de l'Europe, est aussi importante pour le mouvement français de réinvention de l'art public. Plusieurs séries de commandes, près d'un millier au total, sont engagées pour la décoration du Palais de la Découverte (Léger, Herbin, Lipchitz et Laurens) ainsi que pour les nombreux pavillons temporaires. Le Corbusier réalise le Pavillon des Temps modernes, Mallet-Stevens le pavillon de l'Électricité et de la Lumière dont Georges-Henri Pingusson aménage la scénographie lumineuse et le mât qui signale les entrées. Ce dernier édifie aussi le pavillon de l'U.A.M. (Union des artistes modernes), manifeste esthétique du style "paquebot». Notons aussi la décoration créée par Robert et Sonia Delaunay pour le pavillon de l'Air et celui des Chemins de fer.

Conjointement à ces commandes, marquées par une préoccupation d'ouverture aux courants novateurs, Jean Zay jette les bases du « $1 \%$ décoration », fraction du budget global d'une construction publique et destinée à la commande d'une œuvre d'art. Le premier pourcentage, établi à $1,50 \%$, est limité aux projets de construction d'établissements d'enseignement. Citons quelques décorations murales réalisées à cette époque : le Conservatoire des arts et métiers (André Lhote), l'amphithéâtre de l'École de pharmacie (Charles Dufresne) ou encore la ménagerie du Muséum d'histoire naturelle (Raoul Dufy). Cette expérience trahit bien les limites inhérentes à sa première dénomination ( $1 \%$ décoration »). Il faudra attendre l'après-guerre pour que la sculpture publique retrouve un statut d'œuvre d'art à part entière (Cf. infra 2.2).

\section{Le renouveau de la sculpture publique et le programme des œuvres de plein air de La Défense}

8 En continuité avec la politique culturelle du Front Populaire, l'instauration du « $1 \%$ décoration $»^{4}$ est généralisée en 1951 et va réellement permettre le renouveau de la sculpture publique dans le paysage urbain. Rappelons qu'il oblige tout maître d'ouvrage d'un programme immobilier à consacrer cette fraction à la réalisation d'une œuvre d'art originale sous la responsabilité conjointe du maître d'ouvrage et de l'architecte concepteur du programme. Nos édiles ont ainsi rendu possible l'intégration des sculptures contemporaines dans les sites urbains.

\section{La problématique de la Communication-médiation des cultures}

9 La problématique de la Communication-médiation des cultures est présente depuis fort longtemps au sein des SIC (Sciences de l'Information et de la Communication) puisque dans les années 1980 furent généralisés des DEUG intitulés Médiation culturelle et Communication et même Lettres, Arts, Expression, Communication (LAEC - Sorbonne Nouvelle Paris III).

10 Le groupe Cultures et Médiation a été créé lors du X ${ }^{\text {ème }}$ Congrès de la SFSIC ${ }^{5}$ qui se déroula à l'Université de Grenoble-Echirolles en novembre 1996. Il s'est proposé de susciter des manifestations d'échanges et de débats pour les chercheurs ou les enseignants- 
chercheurs engagés dans de tels travaux depuis de nombreuses années pour la plupart d'entre eux. Un de ses thèmes fédérateurs est le constat de l'importance des dimensions informationnelles et communicationnelles présentes dans les diverses expressions de la culture, que celles-ci intègrent les techno-sciences informatiques ou non.

\section{Le colloque Les arts de la ville et leur médiation (Université Paul Verlaine de Metz)}

11 C'est en janvier 2001, lors du XII ${ }^{\text {ème }}$ Congrès de la SFSIC (Palais de l'UNESCO) que j'ai proposé la thématique Les arts de la ville et leur médiation approuvée aussitôt par les membres du groupe. J'y avais donc été chargé de coordonner le prochain colloque du groupe à l'Université de Metz (où j'avais été élu Professeur en mai 2000) pour la fin du printemps 2002. Cette thématique était fondée sur un certain nombre de constats liés à mes propres axes de recherche. La redécouverte du sens de l'architecture et, plus généralement, de tout aménagement spatial. Il en a résulté un rejet du fonctionnalisme réducteur fortement théorisé par des philosophes, et, depuis le dernier quart du XX ${ }^{\text {ème }}$ siècle, un incontestable renouveau de l'architecture, de la géographie urbaine et de tous les arts de la ville. Ce mouvement a été et est toujours accompagné de nombreuses études et recherches (Giulio Carlo Argann, André Chastel ${ }^{8}$, Hubert Damisch, Vittorio Gregotti, Bernard Huet ${ }^{9}$, Christian Norberg-Schulz, Marcel Roncayolo, Aldo Rossi ${ }^{10}$, Manfredo Tafuri ...) et s'est aussi traduit par la réévaluation d'œuvres plus anciennes (Camillo Sitte, Erwin Panofsky, Pierre Francastel ...). Ce mouvement a été et est toujours accompagné de nombreuses études et recherches dont ce colloque a souhaité rendre compte. Le colloque Les Arts de la ville dans la prospective urbaine - Débat public et médiation - intégré à la Semaine de la Ville 2006 (Université François Rabelais De Tours).

\section{Problématique scientifique du colloque et objectifs}

C'est en 2004 que nous nous sommes à nouveau trouvés investis de la tâche d'organiser pour 2006 une nouvelle édition. Le comité de pilotage de la MSH «Villes et territoires » a décidé de l'inclure au sein des manifestations de Semaine de la Ville 2006. En fonction de la thématique choisie avant mon arrivée (Utopies et expérimentations), j'ai proposé l'intitulé : Les Arts de la ville dans la prospective urbaine - Débat public et médiation.

L'espace urbain a toujours été le lieu de la manifestation d'utopies ou d'expérimentations qui sont la traduction de recherches prospectives quant à l'habiter, de la demeure individuelle ou collective aux édifices publics, religieux ou profanes. Ces prospectives dans lesquelles l'esprit et l'art des lieux s'affirment par une forte identité sont aussi souvent liées à des mouvements artistiques et/ou des avant-gardes d'idées. Par définition, elles sont porteuses d'intentions fortes qui suscitent une interrogation sur leurs sens multiples, alimentant un débat public intense. L'argumentaire mis au jour à cette occasion constitue une part importante de leur communication/médiation. Le colloque de Tours s'est proposé de rassembler les travaux qui tentent de donner un éclairage sur les nombreuses questions liées à cette vaste problématique, en respectant leur caractère transdisciplinaire. Cette problématique est abordée au sein des grandes catégories de réalités spatiales qui constituent les Arts de la ville:

1. Architecture et compositions urbaines

2. Sculpture publique - Art dans la ville 
3. Art des jardins - Paysage urbain

4. Arts de la rue et de l'espace public redécouverte de l'importance du sens de l'architecture et, plus généralement, de tout aménagement spatial. Cela s'est traduit par une réinvention de l'art des lieux, de la sculpture publique et de l'art des jardins et des paysages. A ces catégories canoniques des arts de la ville, correspondront les quatre grands axes du colloque. En relation avec le thème fédérateur de la Semaine de la Ville 2006 - Utopies et expérimentations, ont été notamment accueillis les travaux et recherches mettant en relation Les arts de la ville et leurs dimensions prospectives. édition, intégrée à la Semaine de la Ville 2006, est portée par le CEDPM, équipe d'accueil (EA 3253) de la MSH «Villes et Territoires». Elle met donc aussi l'accent sur l'étude du débat public et des processus délibératifs, composantes capitales des dispositifs communication-médiation de tout aménagement spatial. En effet, le patrimoine n'existe dans la conscience collective qu'au moyen de la communication qui en élucide les langages architectoniques et plastiques. De la même façon, toute innovation architecturale et/ou urbaine requiert des dispositifs de débat public et de communication-médiation pour être comprise et approuvée par les citoyens. Un cinquième axe a donc été constitué par les auteurs qui ont choisi d'expliciter des aspects de la médiation spatiale:

\section{Débat public et médiation}

\section{Les cinq axes et le déroulement de leurs ateliers}

Après le développement des problématiques par Pascal Sanson, responsable scientifique de la manifestation, Loïc Vaillant, Vice-président chargé de la Recherche, et Serge Thibault, Directeur de l'UMR CITERES, ont souligné l'importance et l'originalité de la manifestation.

\section{Session Axe 1 : Architecture et compositions urbaines}

Marcel Roncayolo et Pascal Sanson ont été les modérateurs de la session qui a rassemblé neuf contributions :

Giuseppe BONACORSI, «La ville moderne : Identité et qualité urbaine »

Heddya BOULKROUNE, Abdelkader BEN SACI, "Architectures de la rue: processus d'information sémio-morphique "

Patrizia LAUDATI et Hafida BOULEKBACHE, «Prospectives urbaines: de la connaissance des villes aux stratégies d'action "

Isabelle de ROSE, «Le beffroi : symbole iconique d'une identité urbaine et régionale »

Josep MUNTAÑOLA THORNBERG, «Barcelona 2000 - Effets de la globalisation »

Damien MASSON, "Le mouvement: un révélateur des potentialités esthétiques de l'architecture ordinaire?» 

public»

\section{Session Axe 4 : Arts de la rue et de l'espace public} Barcelone " place » rassemblé huit contributions : du monument de l'indépendance de l'Ouzbékistan $»^{11}$

\section{Session Axe 3 : Art des jardins - Paysage urbain} Tuileries » L'utopie de l'urbain à Lisbonne » sept contributions : une recherche. affoulements et créativité de l'espace public »
Luis Angel DOMÍNGUEZ MORENO, "Habiter le lieu: le marché de Sainte-Catherine à

Christian DEHAYNIN et Bernard PAGAND, «Strasbourg: la question de la gare et de sa

Diana PALAZOVA-LEBLEU, « Lille, 1900-1939: La construction d'une ville moderne ».

\section{Session Axe 2 : Sculpture publique - Art dans la ville}

Michelle Sustrac et Sylviane Leprun ont été les modératrices de la session qui a

Bruno LONCHAMPT, « Enjeu de la présence artistique dans l'espace public urbain »

Michel COSTANTINI, "Sculpture publique et environnement : l'exemple de la statue équestre »

Boris CHUKHOVICH, "Symbolisme du globe et mythologie du pouvoir: analyse iconologique

Alexandre CUBIZOLLES et Sabine THUILIER, « Le BULB »

Emmanuelle DUCROCQ, « 'Un autre regard' : installation-performance »

Catherine GROUT, « L'œuvre processus comme mode d'intervention collective »

Gilbert SMADJA, " Art et espace public : pour une démarche urbaine »

Valérie MELIANI, «Art numérique et espace public : quels positionnements ? Quels enjeux?»

Christian Calenge a été le modérateur de la session qui a rassemblé six contributions :

Régis LABOURDETTE, « Le promeneur et la sculpture moderne et contemporaine au Jardin Des

Catarina Luísa Teles Ferreira CAMARINHAS, "De l'avenue-promenade au 'greenway':

Meriem BENKHEDDA MAMMERI, « Couleur et Identité urbaine en Algérie

Robert-Max ANTONI \& Aude VASPART, « Le concours international arturbain.fr »

Marina TRAYSER, "Les Yeux de la Ville', ou l'éphémère comme outil pour transformer l'espace

Nicole Denoit et Corinne Larrue ont été les modératrices de la session qui a rassemblé

Michelle SUSTRAC, « Entre discours et pratiques, nouveaux horizons »

Liane MOZERE, "Arts dans la rue : l'exemple de Sotteville-lès-Rouen »

Maud LE FLOC'H, « Des arts de la rue au projet urbain, "Mission repérage(s), un élu-un artiste",

Paul RASSE \& Nicolas PELISSIER, "Cultures de la post modernité, entre fluidité des

André SAUVAGE, « La fête, révélateur de notre temps... » 


\section{Synthèse des contributions}

\section{Session Axe 5 : Débat public et médiation} dix contributions : patrimoine urbain par les habitants " environnement lisible " les monuments » industriels " régions annexées"

\section{La Table ronde finale}

Hamza ZEGHLACHE, « Espace public, rituel et politique dans la ville arabo-musulmane »

Abdelwahed ALLOUCHE, "Le livre dans la rue : une démarche du 'Système Enfant-Rue ».

Michelle Sustrac et Pascal Sanson ont été les modérateurs de la session qui a rassemblé

Laura GARCIA VITTORIA, «Pour une prospective de la médiation de l'espace public »

Michèle GELLEREAU, Emilie DALAGE, "L'expert et l'amateur : valoriser l'interprétation du

Albert LEVY, « Vers un urbanisme transactionnel ?»

Caroline LENOIR, "Chanter la ville : sémantique et médiation dans l'espace public »

Anne GONON, «Quand est-ce qu'on arrive ? : le spectacle comme espace de débat public »

Valérie VITALBO, "Pour une approche des espaces muséographiques comme un

Daniel JACOBI, « La fréquentation du patrimoine architectural urbain : visiter la ville, Visiter

Natacha CYRULNIK, "Expérimenter l'utopie pour révéler une réalité »

Orla GELAS, «La transmission, la médiation et l'appropriation de la culture des lieux

Wanda WEISSE, «1871-1914: Esquisse d'analyse d'une architecture allemande dans les

Pascal Sanson a introduit et animé la très riche table ronde finale qui a réuni :

Eric LENGEREAU, Chef du Bureau de la Recherche architecturale, urbaine et paysagère.

Josep MUNTAÑOLA THORNBERG, Universitat Polytecnica de Catalunya.

Robert-Max ANTONI, Président du Séminaire Robert Auzelle.

Michelle SUSTRAC, Urbaniste, PUCA (Plan Urbanisme Construction Architecture).

Michel DOLLFUS, Directeur du SDAP d'Indre et Loire.

67 Nous avions communiqué le volume des résumés, distribué à l'occasion de la manifestation tourangelle. Nous joignons au présent rapport la synthèse a posteriori avec l'aide des modératrices et modérateurs des cinq sessions.

\section{Session Axe 1 : Architecture et compositions urbaines}

Cette session a confirmé l'importance de la dimension médiationnelle de l'architecture et des compositions urbaines. Les intervenants ont eu l'occasion d'échanger sur la nécessité de mettre en œuvre des méthodologies d'analyse qui puissent aider à la 
redécouverte du sens de ces architectures, et permettre une évaluation des dispositifs médiationnels existants.

\section{Session Axe 2 : Sculpture publique -Art dans la ville} ont témoigné de la dynamique engagée par les acteurs du patrimoine. A été soulignée souvent l'importance de la fonction esthétique dans tout aménagement spatial, point mis en exergue par l'exposé des motifs du colloque. La question de l'emplacement que Michel Costantini aborde dans sa dimension sémantique et sémiotique n'en est que plus actuelle.

\section{Session Axe 3 : Art des jardins - Paysage urbain}

Les communicants se sont exprimés sur le concept de paysage urbain et sur la nécessité de percevoir la ville comme système de signification(s). Les thématiques abordées ont mis en lumière la relation qui unit les habitants à leur ville, les « langages de la ville » au sens défini par Roland Barthes ${ }^{12}$.

\section{Session Axe 4 : Arts de la rue et de l'espace public}

71 La diversité des performances et des projets présentés conforte l'hypothèse que les arts de la rue participent activement à l'animation de la cité et à sa sémantisation sociale. Les intervenants ont échangé sur la contribution des arts de la rue au développement des espaces publics; ils se sont interrogés sur leur participation positive ou négative au «vivre ensemble».

\section{Session Axe 5 : Débat public et médiation}

Comme proposé dans l'exposé des motifs, les conférenciers se sont exprimés sur l'étude du débat public et des processus délibératifs, composantes capitales des dispositifs communication-médiation de tout aménagement spatial. Chercheurs, acteurs et artistes se sont rejoints sur l'importance de formaliser et de modéliser les réflexions et travaux engagés sur ces problématiques.

\section{Conclusion : Un vaste champ de recherche}

73 Toutes ces contributions et les interventions de la table ronde, émanation d'une cinquantaine de recherches et/ou réflexions, montrent à quel point les arts de la ville sont à présent une composante indispensable à toute prospective urbaine. De même, au $\mathrm{vu}$ de nombreuses recherches présentées, on constate que toute innovation architecturale et/ou urbaine requiert bien des dispositifs de débat public et de communication-médiation pour être comprise et approuvée par les citoyens. 


\section{NOTES}

1. Ce constat a motivé un grand nombre de nos travaux, nous renvoyons à la bibliographie.

2. Dans le cadre des $\mathrm{III}^{\mathrm{e}}$ Journées Internationales de Sémiotique de Blois, sous le haut patronage de M. Jack Lang, alors Ministre de la Culture et Maire de Blois.

3. LE PAYSAGE URBAIN - Représentations, Significations, Communication, (dir. P. Sanson), Editions L'Harmattan (Collection EIDOS), 2007.

4. Cette première dénomination, un peu limitée, laissera place à l'actuelle appellation : « $1 \%$ artistique "

5. Société Française des Sciences de l'Information et de la Communication - SFSIC.

6. Docteur en SIC mais aussi architecte D.P.L.G., nous en sommes l'un des membres fondateur et représentons, avec quelques autres enseignants-chercheurs au sein du groupe, le champ disciplinaire Architecture, urbanisme et paysage.

7. Argan Giulio Carlo, Projet et destin - Art, architecture, urbanisme, 1964 ; tr.fr., Paris, Éd. de la Passion, 1993 ; Histoire de l'art et la ville, Editore Riuniti, 1983 ; tr. fr., Paris, Éd. de la Passion, 1995.

8. Chastel André, Fables, formes, figures, Paris, Flammarion, 1978 ; Architecture et patrimoine, Paris, Imp. Nation., 1994.

9. Huet Bernard, Anachroniques d'architecture, Archives d'Architecture moderne, Bruxelles, 1981; L'Architecture et la ville. Mélanges offerts à Bernard Huet, Éditions du Linteau, Paris, 2000.

10. Rossi Aldo, L'architecture de la ville, Paris, L'Equerre, 1981.

11. Malgré son absence due à des problèmes de visa, Boris Chukhovich nous a promis un article qui figurera dans les actes.

12. Sémiologie et urbanisme, in L'aventure sémiologique, Paris, Seuil, 1985 \& in Barthes - Cuvres complètes, Tome 1, Paris, Seuil, 1993.

\section{AUTEUR}

\section{PASCAL SANSON}

Responsable scientifique

pascal.sanson@univ-tours.fr 